\title{
Dosimetric impact of intermediate dose calculation for optimization convergence error
}

\author{
Byung Do Park ${ }^{1}$, Tae Gyu Kim ${ }^{1}$, Jong Eon Kim² \\ ${ }^{1}$ Department of Radiation Oncology, Samsung Changwon Hospital, Sungkyunkwan University School of Medicine, Changwon, \\ Korea \\ ${ }^{2}$ Department of Radiological Science, Kaya University, Gimhae, Korea
}

Correspondence to: Jong Eon Kim, e-mail: kjepk@kaya.ac.kr

Keywords: intensity modulated radiation therapy (IMRT), intermediate dose calculation, anisotropic analytical algorithm $(A A A)$

Received: January 12, $2016 \quad$ Accepted: February 11, $2016 \quad$ Published: February 26, 2016

ABSTRACT

Intensity-modulated radiation therapy (IMRT) provides the protection of the normal organs and a precise treatment plan through its optimization process. However, the final dose-volume histogram (DVH) obtained by this technique differs from the optimal DVH, owing to optimization convergence errors. Herein, intermediate dose calculation was applied to IMRT plans during the optimization process to solve these issues.

Homogeneous and heterogeneous targets were delineated on a virtual phantom, and the final DVH for the target volume was assessed on the target coverage. The IMRT plans of 30 patients were established to evaluate the usefulness of intermediate dose calculation.

The target coverage results were analogous in the three plans with homogeneous targets. Conversely, conformity indices (conformity index [CI], heterogeneity index $[\mathrm{HI}]$, and uniformity index [UI]) of plans with intermediate dose calculation were estimated to be more homogenous than plans without this option for heterogeneous targets (CI, 0.371 vs. 1.000 ; HI, 0.104 vs. 0.036 ; UI, 1.099 vs. 1.031 for Phantom B; and CI, 0.318 vs. 0.956 ; HI, 0.167 vs. 0.076 ; UI, 1.165 vs. 1.057 for Phantom C). In brain and prostate cancers, a slight difference between plans calculated with anisotropic analytical algorithm (AAA) was observed (HI, $p=0.043, \mathrm{UI}, p=0.043$ for brain; HI, $p=0.042, \mathrm{UI}, p=0.043$ for prostate). All target coverage indices were improved by intermediate dose calculation in lung cancer cases $(p=0.043)$.

In conclusion, intermediate dose calculation in IMRT plans improves the target coverage in the target volume around heterogeneous materials. Moreover, the optimization time can be reduced.

\section{INTRODUCTION}

The development of intensity-modulated radiation therapy (IMRT) has led to significant improvements in dose conformity; however, this technique increases the time needed for the dose calculation and plan optimization. On the contrary, volumetric modulated arc therapy (VMAT) technique developed by Otto reduces the beam delivery time and inter-fractional error by using fewer monitor units than IMRT [1], moreover, VMAT technique improves the target coverage and reduces systematic error in normal organ [2]. Recent progress made in treatment planning technology has led to improvements in the treatment efficiency, as well as to reduced side effects. However, in the radiation treatment planning process, the final dose-volume histogram (DVH) acquired by IMRT or VMAT differs from the optimal DVH obtained via the optimization process. This is caused by inherent errors in the dose calculation algorithm, such as dose calculation for lateral scatter or buildup region errors due to modeling modulator transmission. This issue arises from optimization convergence error, especially in cases of tissue heterogeneity $[3,4]$.

In recent years, several studies have been conducted on the optimization of convergence error. In one previous study, an improved final DVH was achieved by using 
the base dose that was acquired from the initial plan to solve the optimization convergence error [5]. Meanwhile, innovative method, named intermediate dose calculation, has been previously applied to the VMAT technique with the jaw tracking option in Progressive resolution optimizer (PRO3) algorithm [6], it was applied to IMRT plans in Eclipse version 11 (Varian Medical Systems, Palo Alto, CA) for the first time in 2009 by Zacarias and Mills [4].

Intermediate dose calculation is carried out during the optimization of the initial plan, after which optimization is continuously performed with the calculated dose distribution; subsequently, the dose calculation algorithm is applied to the treatment plan once more after the optimization is finished. Intermediate dose calculation is essential to ensure an improved and rapid optimization process and target coverage. In addition, for accurately calculating the optimization for heterogeneous materials, Acuros XB (AXB), a specific dose calculation algorithm, was developed [7]. AXB algorithm is similar to classic Monte Carlo method for calculating of dose distribution in heterogeneous material, on the other hand, anisotropic analytical algorithm (AAA) is the dose calculation algorithm base on a pencil beam convolution-superposition method [8]. AAA algorithm in dose calculation improved the weakness of pencil beam convolution algorithm in heterogeneous material. However this algorithm still has the dosimetric error in soft tissue. Whereas, several studies reported that $\mathrm{AXB}$ is superior to AAA in inhomogeneous and heterogeneous material [9-11].

This study aimed to investigate the dosimetric impact of intermediate dose calculation by DVHs, and we moreover briefly examine the dosimetric differences between AAA and AXB algorithms.

\section{RESULTS}

\section{Phantom study}

All results derived from the phantom study are summarized in Table 1. The target coverages investigated with Phantom A showed similar results, and the maximum doses for the target volumes were $102.6 \%, 101.8 \%$, and $102.6 \%$ in Plan1, Plan1-DC, and Plan1-int, respectively. On the other hand, the maximum doses were increased in Phantoms B and $\mathrm{C}$, which included heterogeneous materials. The maximum doses in Phantom B was $99.7 \%$, 101.6\%, and 101.8\%, respectively, in Plan1, Plan1-DC, and Plan1-int, while the maximum doses in Phantom C were $99.2 \%, 102.8 \%$, and $103.9 \%$, respectively. As a result, we achieved measurement results with non-negligible differences for the target coverage compared to those in Phantom A.

DVH results of the target volume are plotted in Figure 1. DVHs of all plans in Phantom A (Figure 1. (a)) showed similar results, whereas DVH results for the target volume in Plan1-DC and Plan1-int were significantly improved by using intermediate dose calculation (Figure 1. (b) and (c)).

\section{Clinical cases}

Figure 2 shows DVHs of example cases in each treatment region. The dose distributions were calculated by AAA and AXB algorithms. The target coverage was analyzed by DVHs for 30 cancer cases and evaluated by the mean value and standard deviation of CI, HI and UI which are measured on treatment planning system.

All results calculated by AAA are summarized in Table 2. As a result, in the case of $\mathrm{HN}$, no clinical difference was found. On the other hand, significant clinical differences were observed for the HI $(p=0.043)$ and UI ( $p=0.043)$ between Plan1 and Plan1-int in brain cancer cases, and for HI $(p=0.042)$ between Plan1 and Plan1- DC and UI ( $p=0.043)$ between Plan1 and Plan1-int in prostate cancer cases. Table 3 shows the results obtained by AXB. This result showed a similar tendency to those obtained by AAA. In the case of lung cancer, considerable differences in both AAA and AXB algorithms were observed for the target coverage upon intermediate dose calculation, indicating that DVH can be achieved accurately by intermediate dose calculation for the treatment target around heterogeneous material.

The dose maximum values by AAA and AXB algorithms for the target volume are plotted in Figure 3. In Plan1-DC, the average maximum values by AAA were $110.4 \%, 113.3 \%, 103.4 \%$, and $108.6 \%$ in $\mathrm{HN}$, brain, prostate, and lung cancer cases, respectively. The corresponding average maximum values by AXB were $110.7 \%, 113.0 \%, 104.8 \%$, and $107.2 \%$, respectively. These results were higher than those for Plan1 and Plan1-int.

Figure 4 shows the target coverage calculated by AAA and AXB algorithms in lung cancer cases. The result revealed that dose distribution by using intermediate dose calculation showed better target coverage in both AAA and AXB algorithms. A comparative study of the dosimetric impact on AAA and AXB algorithms for lung cancer cases are illustrated in Table 4.

A minor relative difference was observed between AAA and AXB algorithms, indicating that the target coverage was lower in AXB plans than in AAA. In Plan1, significant differences between AAA and AXB algorithms for $\mathrm{D}_{98 \%}, \mathrm{D}_{2 \%}$, and $\mathrm{D}_{\text {mean }}$ were observed, whereas these significant differences were reduced between Plan1-DC and Plan1-int. These results were similar to the values observed in the other regions (Table 5).

\section{DISCUSSION}

Treatment plans using IMRT and VMAT techniques are associated with a precise target volume and minimized side effects due to enhanced protection of the normal organ. However, many trials are currently required to 
Table 1: Comparisons of the target coverage in each phantom

\begin{tabular}{|c|c|c|c|c|}
\hline & & Plan1 & Plan1-DC & Plan1-int \\
\hline \multirow{3}{*}{ Phantom A } & CI & 1.000 & 1.000 & 1.000 \\
\hline & HI & 0.030 & 0.026 & 0.032 \\
\hline & UI & 1.026 & 1.023 & 1.028 \\
\hline \multirow{3}{*}{ Phantom B } & CI & 0.371 & 1.000 & 1.000 \\
\hline & $\mathrm{HI}$ & 0.104 & 0.039 & 0.036 \\
\hline & UI & 1.099 & 1.034 & 1.031 \\
\hline \multirow{3}{*}{ Phantom C } & $\mathrm{CI}$ & 0.318 & 0.930 & 0.956 \\
\hline & $\mathrm{HI}$ & 0.167 & 0.070 & 0.076 \\
\hline & UI & 1.165 & 1.051 & 1.057 \\
\hline
\end{tabular}

CI: conformity index, HI: heterogeneity index, UI: uniformity index.
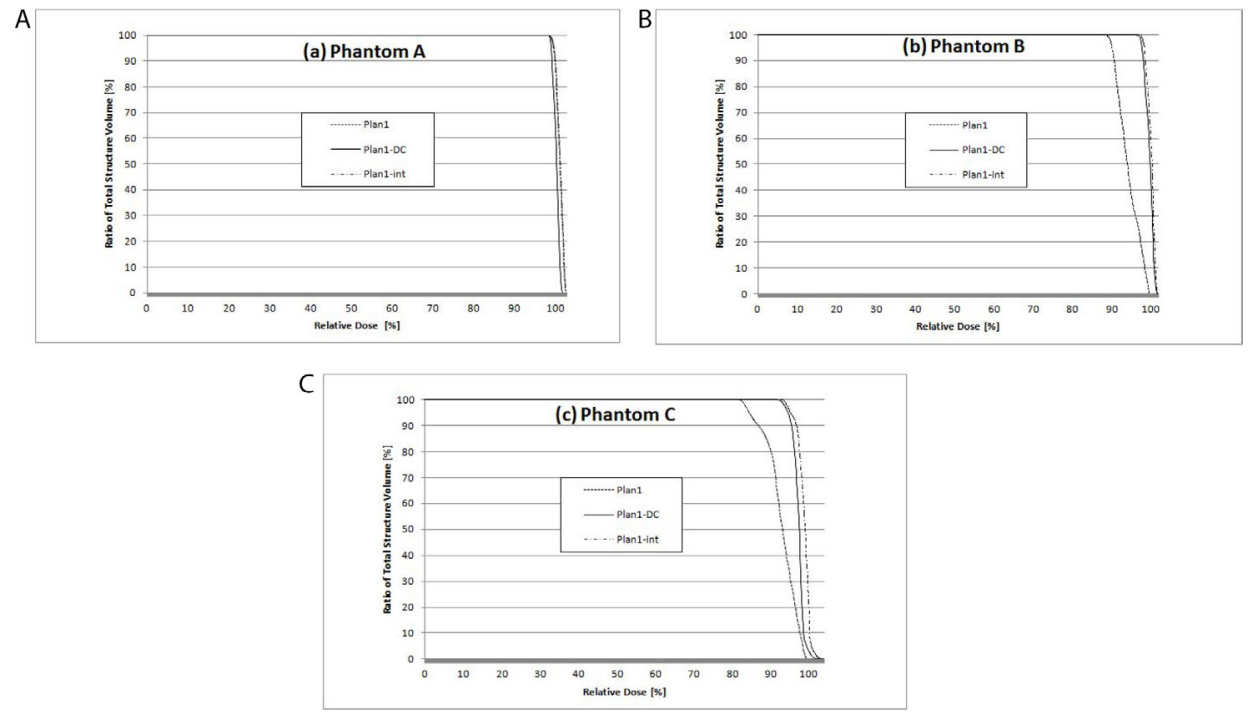

Figure 1: Dose volume histograms of the target volume in each phantom. (a) Phantom A, (b) Phantom B, and (c) Phantom C. Dotted line $=$ plan1; solid line $=$ plan1-DC; dot-and- dashed line $=$ plan1-int.
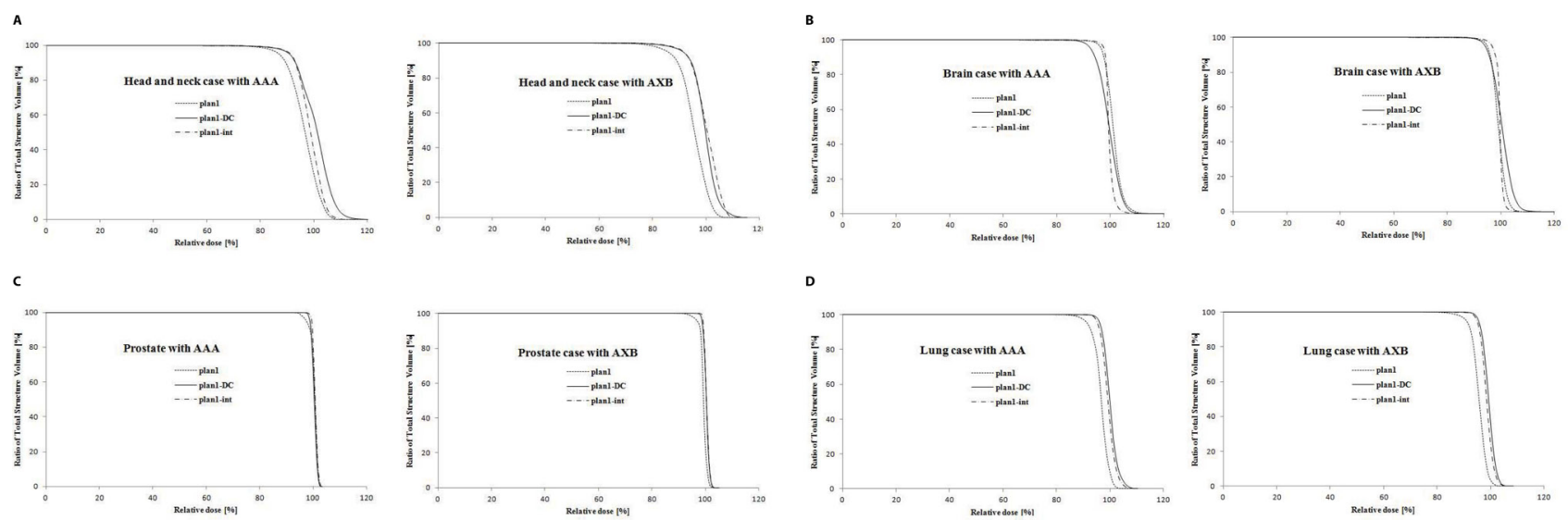

Figure 2: Dose-volume histograms of the target volumes calculated with anisotropic analytic algorithm (AAA) and Acuros XB (AXB) algorithm in Plan1, Plan1-DC, and Plan1-int for head and neck, brain, prostate, and lung cancer cases. Dotted line $=$ plan 1 ; solid line $=$ plan1-DC; dot-and-dashed line $=$ plan 1 -int. 
Table 2: Summary of each parameter measured by AAA for each region

\begin{tabular}{|c|c|c|c|c|c|c|}
\hline \multirow{2}{*}{\multicolumn{2}{|c|}{$\mathbf{A A A}$}} & \multirow[b]{2}{*}{ Plan1 } & \multirow[b]{2}{*}{ Plan1-DC } & \multirow[b]{2}{*}{ Plan1-int } & \multicolumn{2}{|c|}{$p$-value } \\
\hline & & & & & $\begin{array}{l}\text { Plan1 vs. } \\
\text { Plan1-DC }\end{array}$ & $\begin{array}{l}\text { Plan1 vs. } \\
\text { Plan1-int }\end{array}$ \\
\hline \multicolumn{7}{|c|}{ Head and neck } \\
\hline \multirow{2}{*}{ CI } & Mean & 0.833 & 0.913 & 0.833 & \multirow{2}{*}{0.221} & \multirow{2}{*}{0.925} \\
\hline & SD & 0.221 & 0.084 & 0.190 & & \\
\hline \multirow{2}{*}{ HI } & Mean & 0.126 & 0.139 & 0.108 & \multirow{2}{*}{0.320} & \multirow{2}{*}{0.073} \\
\hline & SD & 0.086 & 0.090 & 0.085 & & \\
\hline \multirow{2}{*}{ UI } & Mean & 1.099 & 1.117 & 1.083 & \multirow{2}{*}{0.132} & \multirow{2}{*}{0.147} \\
\hline & $\mathrm{SD}$ & 0.060 & 0.073 & 0.061 & & \\
\hline \multicolumn{7}{|l|}{ Brain } \\
\hline \multirow{2}{*}{ CI } & Mean & 0.981 & 0.967 & 0.973 & \multirow{2}{*}{0.715} & \multirow{2}{*}{0.273} \\
\hline & $\mathrm{SD}$ & 0.017 & 0.052 & 0.030 & & \\
\hline \multirow{2}{*}{ HI } & Mean & 0.094 & 0.096 & 0.077 & \multirow{2}{*}{0.892} & \multirow{2}{*}{0.043} \\
\hline & SD & 0.031 & 0.049 & 0.023 & & \\
\hline \multirow{2}{*}{ UI } & Mean & 1.072 & 1.077 & 1.058 & \multirow{2}{*}{0.893} & \multirow{2}{*}{0.043} \\
\hline & $\mathrm{SD}$ & 0.023 & 0.042 & 0.019 & & \\
\hline \multicolumn{7}{|l|}{ Prostate } \\
\hline \multirow{2}{*}{ CI } & Mean & 0.974 & 1.000 & 1.000 & \multirow{2}{*}{0.068} & \multirow{2}{*}{0.068} \\
\hline & SD & 0.032 & 0.000 & 0.000 & & \\
\hline \multirow{2}{*}{$\mathrm{HI}$} & Mean & 0.066 & 0.043 & 0.031 & \multirow{2}{*}{0.042} & \\
\hline & $\mathrm{SD}$ & 0.016 & 0.005 & 0.010 & & $0.0+3$ \\
\hline & Mean & 1.053 & 1.036 & 1.024 & & \\
\hline (1) & SD & 0.016 & 0.005 & 0.008 & $0.04 J$ & 0.042 \\
\hline Lung & & & & & & \\
\hline & Mean & 0.647 & 0.982 & 0.961 & 0042 & 0042 \\
\hline & SD & 0.305 & 0.023 & 0.036 & & \\
\hline & Mean & 0.161 & 0.088 & 0.085 & & \\
\hline 111 & SD & 0.043 & 0.023 & 0.022 & נדרט & נדרט \\
\hline & Mean & 1.140 & 1.070 & 1.069 & & \\
\hline 01 & SD & 0.052 & 0.019 & 0.019 & נדט. & נד.ט \\
\hline
\end{tabular}

AAA: anisotropic analytical algorithm, CI: conformity index, HI: heterogeneity index, UI: uniformity index, SD: standard deviation.

achieve an effective treatment plan during the optimization process, and this is associated with a high rate of error.

This study assessed the dosimetric impact of intermediate dose calculation applied to IMRT technique using Eclipse version 11. Our study revealed that intermediate dose calculation is a useful function for IMRT plans. Especially, the target coverage was improved for target volumes using the intermediated calculation method.
In the study by Zacarias and Mills, the final dose distribution was found to be consistent with the result of the repeated optimization process based on the original plan, and the authors therefore concluded that the intermediate dose option is reasonable [4]. Further, Kan et al. reported that there was no difference according to the application of intermediate dose calculation in their phantom study. However, the authors used VMAT technique and evaluated the results according 
Table 3: Summary of each parameter measured by AXB algorithm for each region

\begin{tabular}{|c|c|c|c|c|c|c|}
\hline \multirow{2}{*}{\multicolumn{2}{|c|}{$\mathbf{A X B}$}} & \multirow[b]{2}{*}{ Plan1 } & \multirow[b]{2}{*}{ Plan1-DC } & \multirow[b]{2}{*}{ Plan1-int } & \multicolumn{2}{|c|}{$p$-value } \\
\hline & & & & & $\begin{array}{l}\text { Plan1 vs. } \\
\text { Plan1-DC }\end{array}$ & $\begin{array}{l}\text { Plan1 vs. } \\
\text { Plan1-int }\end{array}$ \\
\hline \multicolumn{7}{|c|}{ Head and neck } \\
\hline \multirow{2}{*}{ CI } & Mean & 0.778 & 0.923 & 0.779 & \multirow{2}{*}{0.010} & \multirow{2}{*}{0.826} \\
\hline & SD & 0.256 & 0.071 & 0.242 & & \\
\hline \multirow{2}{*}{$\mathrm{HI}$} & Mean & 0.125 & 0.137 & 0.118 & \multirow{2}{*}{0.443} & \multirow{2}{*}{0.426} \\
\hline & SD & 0.075 & 0.084 & 0.087 & & \\
\hline \multirow{2}{*}{ UI } & Mean & 1.100 & 1.109 & 1.095 & \multirow{2}{*}{0.510} & \multirow{2}{*}{0.495} \\
\hline & SD & 0.060 & 0.063 & 0.067 & & \\
\hline \multicolumn{7}{|l|}{ Brain } \\
\hline \multirow{2}{*}{$\mathrm{CI}$} & Mean & 0.940 & 0.978 & 0.959 & \multirow{2}{*}{0.138} & \multirow{2}{*}{0.138} \\
\hline & SD & 0.058 & 0.033 & 0.040 & & \\
\hline \multirow{2}{*}{$\mathrm{HI}$} & Mean & 0.092 & 0.095 & 0.084 & \multirow{2}{*}{0.715} & \multirow{2}{*}{0.465} \\
\hline & $\mathrm{SD}$ & 0.025 & 0.040 & 0.030 & & \\
\hline \multirow{2}{*}{ UI } & Mean & 1.072 & 1.076 & 1.063 & \multirow{2}{*}{0.892} & \multirow{2}{*}{0.285} \\
\hline & SD & 0.021 & 0.035 & 0.024 & & \\
\hline \multicolumn{7}{|l|}{ Prostate } \\
\hline \multirow{2}{*}{$\mathrm{CI}$} & Mean & 0.975 & 1.000 & 0.999 & \multirow{2}{*}{0.043} & \multirow{2}{*}{0.043} \\
\hline & SD & 0.026 & 0.000 & 0.001 & & \\
\hline \multirow{2}{*}{$\mathrm{HI}$} & Mean & 0.068 & 0.044 & 0.034 & \multirow{2}{*}{0.043} & 0012 \\
\hline & $\mathrm{SD}$ & 0.011 & 0.004 & 0.008 & & 0.043 \\
\hline I & Mean & 1.051 & 1.035 & 1.027 & 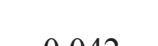 & $0 \Omega 1$ \\
\hline & SD & 0.010 & 0.004 & 0.006 & 0.042 & 0.043 \\
\hline Lung & & & & & & \\
\hline CT & Mean & 0.546 & 0.952 & 0.953 & 042 & 0012 \\
\hline & SD & 0.294 & 0.062 & 0.049 & & \\
\hline IU & Mean & 0.172 & 0.093 & 0.089 & $0 \Omega$ & 0040 \\
\hline 111 & $\mathrm{SD}$ & 0.059 & 0.028 & 0.026 & 0.045 & 0.045 \\
\hline JI & Mean & 1.144 & 1.077 & 1.072 & 0043 & 0042 \\
\hline U1 & $\mathrm{SD}$ & 0.067 & 0.025 & 0.021 & $0.04 J$ & 0.045 \\
\hline
\end{tabular}

AXB: Acuros XB, CI: conformity index, HI: heterogeneity index, UI: uniformity index, SD: standard deviation.

to application of an air cavity correction option simultaneously with the intermediate dose option [20]. $\mathrm{Li}$ et al. presented the effect of the intermediate dose calculation for lung cancer cases. They reported that dose homogeneity and optimization efficiency were improved by utilizing the intermediate dose calculation [21].

Various dose calculation algorithm have been developed to ensure accurate dose calculation. Fogliata et al. reported that $\mathrm{AXB}$ algorithm can be an alternative to the Monte Carlo calculation [9], and Kan et al. demonstrated that AAA may result in overestimations in small fields [22]. Further, it has been reported that in treatment plans with IMRT or VMAT technique, there is a difference between AAA and AXB algorithms [23].

In the present study, we evaluated the adequacy of intermediate dose calculation for four kinds of treatment regions using IMRT technique. Despite the limited number of clinical cases, this function was found to be effective 

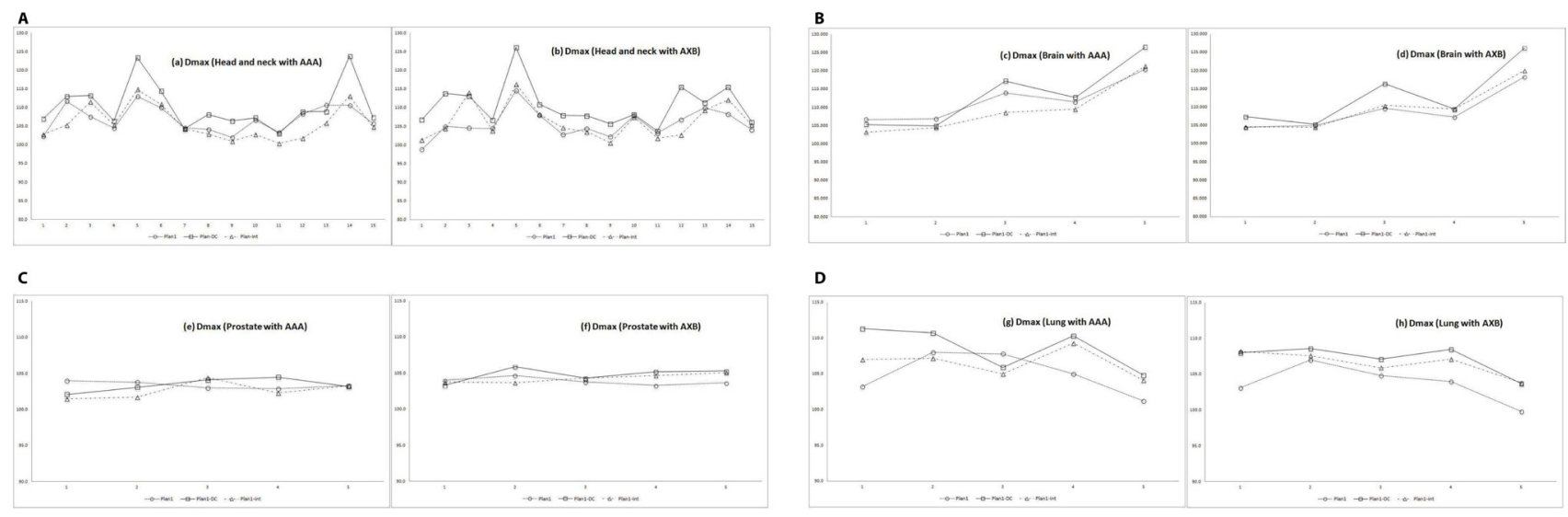

Figure 3: Maximum dose distributions for the target volumes in each region. Dotted line = plan1; solid line = plan1-DC; dot-and-dashed line $=$ plan1-int. The y-axis shows the dose maximum value $(\mathrm{Dmax})$.
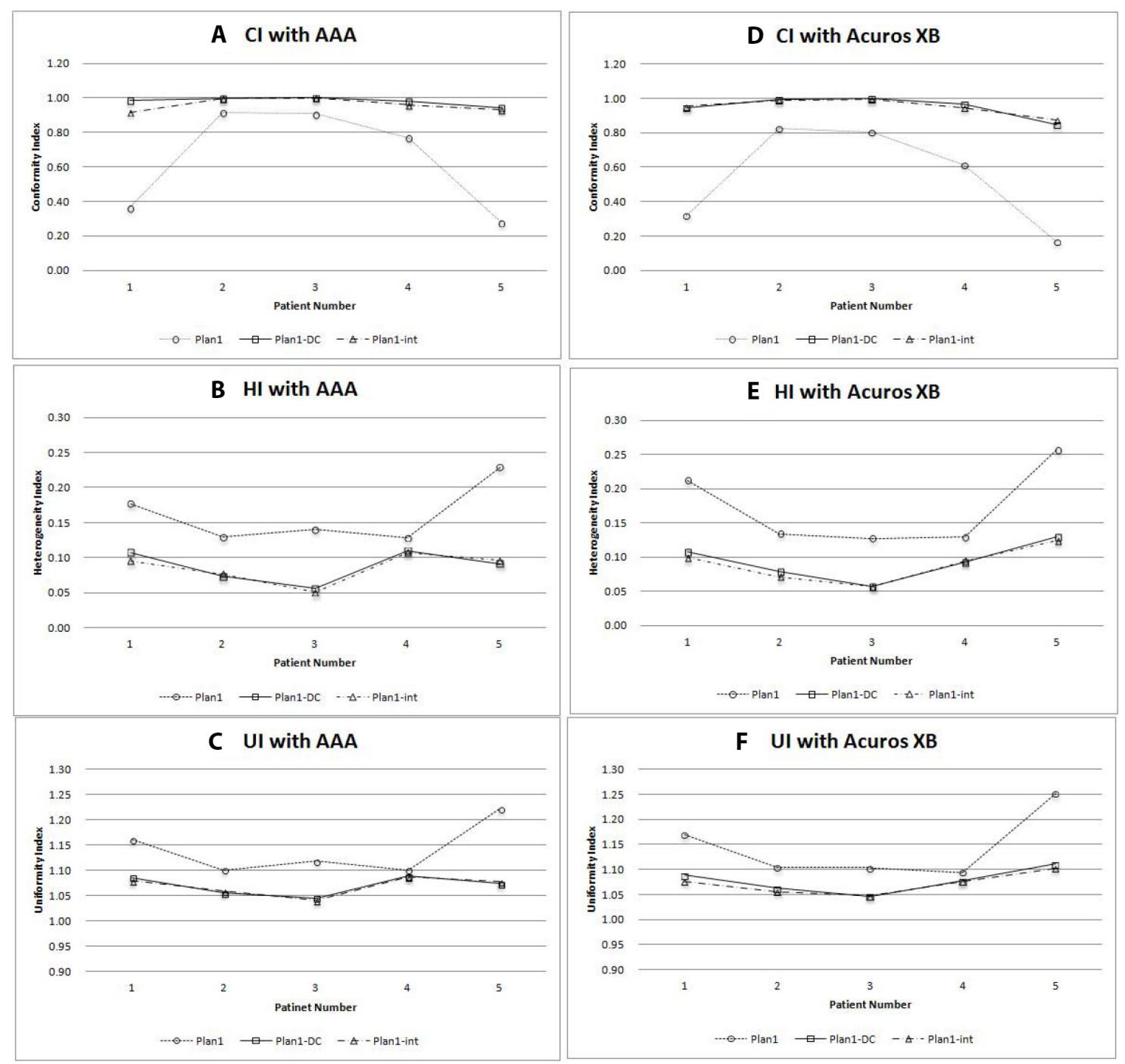

Figure 4: Comparison of conformity index (CI), heterogeneity index (HI), and uniformity index (UI) in each plan calculated by anisotropic analytic algorithm (AAA) (A, B, C), and Acuros XB (AXB) algorithm (D, E, F) for 5 lung cancer cases. Dotted line $=$ plan 1 ; solid line $=$ plan 1 -DC; dot-and-dashed line $=$ plan1-int. 
TABLE 4: AAA vs. AXB for 5 lung cancer cases

Target volume Relative difference (\%) $\quad p$-value Lung volume $\quad$ Relative difference $(\%) \quad p$-value

\begin{tabular}{|c|c|c|c|c|c|c|}
\hline \multirow{4}{*}{ Plan1 } & D98\% & -2.73 & 0.043 & V5Gy & 1.16 & 0.500 \\
\cline { 2 - 7 } & D2\% & -1.44 & 0.043 & V20Gy & -1.64 & 0.138 \\
\cline { 2 - 7 } & Dmean & -1.21 & 0.042 & MLD & -0.47 & 0.357 \\
\hline \multirow{4}{*}{ Plan1-DC } & D98\% & -1.61 & 0.080 & V5Gy & 2.78 & 0.043 \\
\cline { 2 - 7 } & D2\% & -1.11 & 0.080 & V20Gy & -0.12 & 0.893 \\
\hline \multirow{3}{*}{ Plan1-int } & Dmean & -0.88 & 0.080 & MLD & 0.53 & 0.257 \\
\cline { 2 - 7 } & D98\% & -1.04 & 0.080 & V5Gy & 2.33 & 0.225 \\
\cline { 2 - 7 } & D2\% & -0.60 & 0.138 & V20Gy & -0.33 & 0.686 \\
\hline
\end{tabular}

AAA: anisotropic analytical algorithm, AXB: Acuros XB, $\mathrm{D}_{2 / 98 \%}$ : average dose covering $2 \%$ or $98 \%$ of the target volume, $\mathrm{V}_{5 / 20 \mathrm{~Gy}}$ : average lung volume receiving 5 Gy or 20 Gy, MLD: mean lung dose. Relative difference: $(\mathrm{AXB}-\mathrm{AAA}) / \mathrm{AAA} \times 100$.

TABLE 5: AAA vs. AXB for head and neck, brain, and prostate cancer cases (Relative difference)

\begin{tabular}{|c|c|c|c|c|}
\hline & \multirow{2}{*}{ Target volume } & HN & Brain & Prostate \\
\hline & & Relative difference $(\%)$ & Relative difference $(\%)$ & Relative difference $(\%)$ \\
\hline \multirow{3}{*}{ Plan1 } & $\mathrm{D}_{98 \%}$ & -1.34 & -2.48 & -0.60 \\
\hline & $\mathrm{D}_{2 \%}$ & -1.67 & -2.59 & -0.41 \\
\hline & $\mathrm{D}_{\text {mean }}$ & -1.53 & -1.77 & -0.74 \\
\hline \multirow{3}{*}{ Plan1-DC } & $\mathrm{D}_{98 \%}$ & -0.96 & 0.47 & -0.27 \\
\hline & $\mathrm{D}_{2 \%}$ & -0.87 & 0.37 & -0.21 \\
\hline & $\mathrm{D}_{\text {mean }}$ & -0.05 & 0.26 & -0.24 \\
\hline \multirow{3}{*}{ Plan1-int } & $\mathrm{D}_{98 \%}$ & -0.60 & -1.18 & -0.28 \\
\hline & $\mathrm{D}_{2 \%}$ & -0.03 & -0.37 & 0.05 \\
\hline & $\mathrm{D}_{\text {mean }}$ & -0.40 & -0.22 & -0.18 \\
\hline
\end{tabular}

AAA: anisotropic analytical algorithm, AXB: Acuros XB algorithm, $\mathrm{D}_{2 / 98 \%}$ : average dose covering $2 \%$ or $98 \%$ of the target volume, $\mathrm{V}_{5 / 20 \mathrm{~Gy}}$ : average lung volume receiving 5 Gy or $20 \mathrm{~Gy}$, MLD: mean lung dose, HN: head and neck. Relative difference $=(\mathrm{AXB}-\mathrm{AAA}) / \mathrm{AAA} \times 100$.

in obtaining an accurate final DVH. Finally, this study also proposed which dose calculation algorithm should be considered for obtaining a better dose distribution for heterogeneous media through analysis between AAA and $\mathrm{AXB}$ algorithms with intermediate dose calculation.

\section{CONCLUSIONS}

This study analyzed DVHs according to the application of intermediate dose calculation. In conclusion, we found that intermediate dose calculation in IMRT plans improved the target coverage in target volumes surrounded by heterogeneous materials such as target volume in prostate and lung cancer cases. Moreover, the optimization time can be reduced during the optimization process. Therefore, it seems reasonable to conclude that intermediate dose calculation should be applied to IMRT plans. However, the full impact of optimization convergence error remains to be investigated. In the comparison of the differences in dose distribution between AAA and AXB algorithms, our results suggest that the dose calculation should be considered by using the developed AXB algorithm. 


\section{MATERIALS AND METHODS}

\section{Phantom study}

It is necessary to evaluate the dosimetric impact of intermediate dose calculation with a phantom. First, we created three kinds of virtual water phantoms in the treatment planning system: Phantoms A, B, and C. The target volume was created with a diameter of $2 \mathrm{~cm}$ in homogeneous Phantom A. An air cavity, with a diameter of $4 \mathrm{~cm}$, was formed in Phantom B, with the diameter of the target volume in Phantom B being the same as that of Phantom A, but including part of the air cavity. In Phantom $\mathrm{C}$, the target volume was delineated with a diameter of $2 \mathrm{~cm}$ in the center, and a ring with a width of $2 \mathrm{~cm}$ was filled around the target volume (Figure 5). A treatment plan using $6 \mathrm{MV}$ photons with 9 fields was generated for each phantom, and the fields were equally spaced at $40 \circ$ intervals. IMRT technique was applied to all plans, and dose calculation was performed by AAA.

To analyze the dosimetric impact of the intermediate dose calculation, three kinds of treatment plans were established for each phantom. The first plan was created by optimization and dose calculation without intermediate dose calculation (Plan1). The second plan was generated by re-optimizing and re-calculating Plan1 (Plan1-DC). The dose distribution of Plan1 was used as the intermediate dose at this time. The third plan was produced by optimization and dose calculation with intermediate dose calculation from the beginning (Plan1-int).

The target volume coverage was evaluated by the conformity, heterogeneity, and uniformity, using DVHs in Eclipse. Conformity index (CI), heterogeneity index (HI), and uniformity index (UI) were defined as follows [12] :

$$
C I=\frac{T V_{95 \%}}{T V},
$$

where $\mathrm{TV}$ is the target volume and $\mathrm{TV}_{95 \%}$ is the volume covered by $95 \%$ of the prescribed dose;

$$
H I=\frac{D_{2 \%}-D_{98 \%}}{D_{50 \%}},
$$

where $\mathrm{D}_{2 \%}$ is the dose of $2 \%$ of the target volume, $\mathrm{D}_{98 \%}$ is the dose of $98 \%$ of the target volume, and $\mathrm{D}_{50 \%}$ is the dose of $50 \%$ of the target volume [13]; and

$$
U I=\frac{D_{5 \%}}{D_{95 \%}},
$$

where $\mathrm{D}_{5 \%}$ is the dose of $5 \%$ of the target volume, and $\mathrm{D}_{95 \%}$ is the dose of $95 \%$ of the target volume [14].

\section{Clinical cases}

In head and neck (HN) and brain cancer cases, the treatment target volume is complex, and critical normal organs such as the parotid grand, optic apparatus, and spinal cord are adjacent to the target volume. Therefore, a sharp dose response curve and precise dose calculation are required. In a previous study, IMRT technique showed excellent results for these cases in comparison with threedimensional conformal radiation therapy $[15,16]$. In addition, IMRT technique has also been applied to the treatment plan in order to improve the treatment toxicity and the disease-free survival in prostate cancer cases $[17,18]$, while, in lung cancer cases, side effects such as pneumonia can be reduced by protecting the healthy lung, esophagus, heart, and spinal cord using this technique [19]. Thus, using IMRT technique in the treatment plan can also improve the dose escalation for these treatment regions.

In this study, CT scans of 30 patients were randomly chosen to assess the dosimetric impact of intermediate dose calculation. The treatment regions of these patients were in the $\mathrm{HN}(n=15)$, brain $(n=5)$, prostate $(n=5)$, and lung $(n=5)$. The images were obtained with $2.5 \mathrm{~mm}$ slice thickness from 3D-computed tomography (GE Health- care, Buckinghamshire, UK). Three kinds of treatment plans were generated for each image, similar to
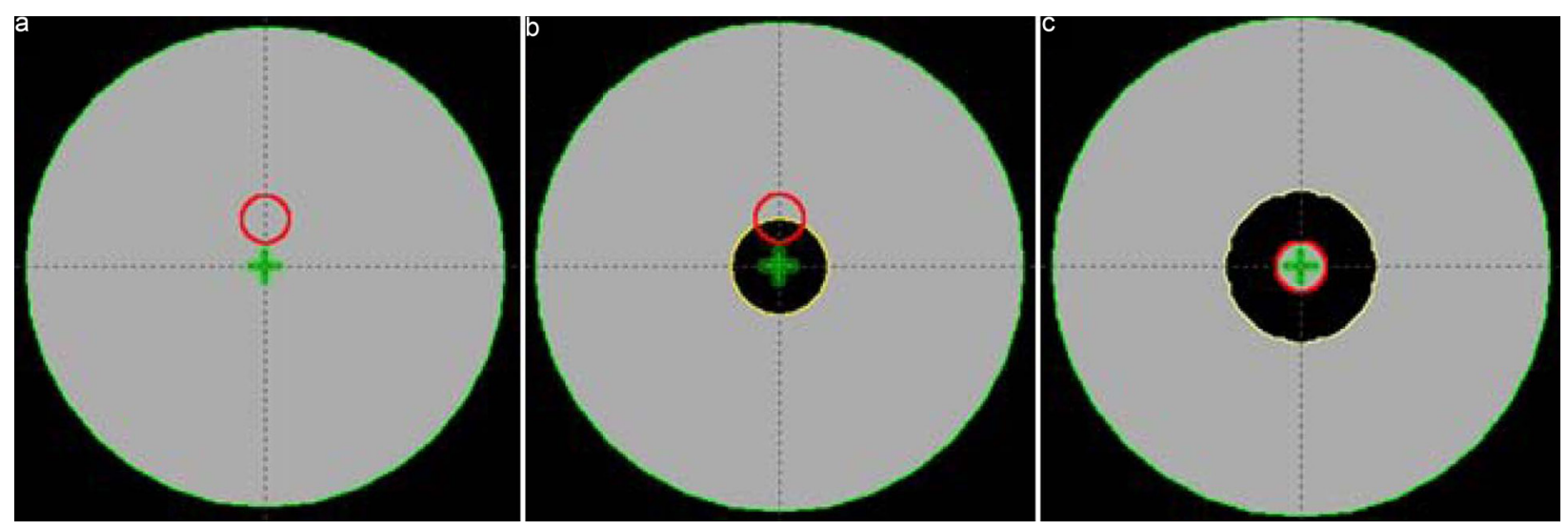

Figure 5: Axial views of the central slice for (a) Phantom A (homogeneous material), (b) Phantom B (small air cavity), and (c) Phantom C (air cavity around the target). 
in the phantom study. The same constraints and priorities in the optimization process were applied to the set of plans (Plan1, Plan1-DC, and Plan1-int). Dose calculation of these treatment plans was carried out by AAA and AXB algorithms.

To evaluate the dosimetric impact of intermediate dose calculation, the target coverage for IMRT plans was estimated by $\mathrm{CI}, \mathrm{HI}$, and UI through DVH. The dose distribution by AAA was compared with that by AXB for each treatment region. For the statistical analyses, the Statistical Program for Social Sciences (SPSS version 18; SPSS Inc., Chicago, IL) was used.

\section{ACKNOWLEDGMENTS AND FUNDING}

We are thankful to our colleagues who provided expertise that greatly assisted the research.

\section{CONFLICTS OF INTEREST}

The authors have no conflicts of interest to declare.

\section{REFERENCES}

1. Otto K. Volumetric modulated arc therapy: IMRT in a single gantry arc. Medical physics. 2008; 35:310-317.

2. Cozzi L, Dinshaw KA, Shrivastava SK, Mahantshetty U, Engineer R, Deshpande DD, Jamema SV, Vanetti E, Clivio A, Nicolini G, Fogliata A. A treatment planning study comparing volumetric arc modulation with RapidArc and fixed field IMRT for cervix uteri radiotherapy. Radiotherapy and oncology. 2008; 89:180-191.

3. Mihaylov IB, Siebers JV. Evaluation of dose prediction errors and optimization convergence errors of deliverablebased head-and-neck IMRT plans computed with a superposition/convolution dose algorithm. Medical physics. 2008; 35:3722-3727.

4. Zacarias AS, Mills MD. Algorithm for correcting optimization convergence errors in Eclipse. Journal of applied clinical medical physics/American College of Medical Physics. 2009; 10:3061.

5. Ong CL, Verbakel WF, Cuijpers JP, Slotman BJ, Lagerwaard FJ, Senan S. Stereotactic radiotherapy for peripheral lung tumors: a comparison of volumetric modulated arc therapy with 3 other delivery techniques. Radiotherapy and oncology. 2010; 97:437-442.

6. Vanetti E, Nicolini G, Nord J, Peltola J, Clivio A, Fogliata A, Cozzi L. On the role of the optimization algorithm of RapidArc((R)) volumetric modulated arc therapy on plan quality and efficiency. Medical physics. 2011; 38:5844-5856.

7. Vassiliev ON, Wareing TA, McGhee J, Failla G, Salehpour MR, Mourtada F. Validation of a new gridbased Boltzmann equation solver for dose calculation in radiotherapy with photon beams. Physics in medicine and biology. 2010; 55:581-598.
8. Tillikainen L, Helminen H, Torsti T, Siljamaki S, Alakuijala J, Pyyry J, Ulmer W. A 3D pencil-beam-based superposition algorithm for photon dose calculation in heterogeneous media. Physics in medicine and biology. 2008; 53:3821-3839.

9. Fogliata A, Nicolini G, Clivio A, Vanetti E, Cozzi L. Dosimetric evaluation of Acuros XB Advanced Dose Calculation algorithm in heterogeneous media. Radiation oncology. 2011; 6:82.

10. Han T, Mourtada F, Kisling K, Mikell J, Followill D, Howell R. Experimental validation of deterministic Acuros $\mathrm{XB}$ algorithm for IMRT and VMAT dose calculations with the Radiological Physics Center's head and neck phantom. Medical physics. 2012; 39:2193-2202.

11. Hoffmann L, Jorgensen MB, Muren LP, Petersen JB. Clinical validation of the Acuros $\mathrm{XB}$ photon dose calculation algorithm, a grid-based Boltzmann equation solver. Acta oncologica. 2012; 51:376-385.

12. Feuvret L, Noel G, Mazeron JJ, Bey P. Conformity index: a review. International journal of radiation oncology, biology, physics. 2006; 64:333-342.

13. Prescribing, Recording, and Reporting Photon-Beam Intensity-Modulated Radiation Therapy (IMRT): Contents. Journal of the ICRU. 2010; 10:NP.

14. Sheng K, Molloy JA, Larner JM, Read PW. A dosimetric comparison of non-coplanar IMRT versus Helical Tomotherapy for nasal cavity and paranasal sinus cancer. Radiotherapy and oncology. 2007; 82:174-178.

15. Mendenhall WM, Amdur RJ, Palta JR. Intensity-modulated radiotherapy in the standard management of head and neck cancer: promises and pitfalls. Journal of clinical oncology. 2006; 24:2618-2623.

16. MacDonald SM, Ahmad S, Kachris S, Vogds BJ, DeRouen M, Gittleman AE, DeWyngaert K, Vlachaki MT. Intensity modulated radiation therapy versus three-dimensional conformal radiation therapy for the treatment of high grade glioma: a dosimetric comparison. Journal of applied clinical medical physics/American College of Medical Physics. 2007; 8:47-60.

17. Ashman JB, Zelefsky MJ, Hunt MS, Leibel SA, Fuks Z. Whole pelvic radiotherapy for prostate cancer using 3D conformal and intensity-modulated radiotherapy. International journal of radiation oncology, biology, physics. 2005; 63:765-771.

18. Edlund T, Zimmer JR, Gannett DE. IMRT for the treatment of prostate cancer: a comparison of a forward-planned technique and an inverse-planned technique utilizing a dose gradient method. Medical dosimetry. 2004; 29:128-133.

19. Christian JA, Bedford JL, Webb S, Brada M. Comparison of inverse-planned three-dimensional conformal radiotherapy and intensity-modulated radiotherapy for non-small-cell lung cancer. International journal of radiation oncology, biology, physics. 2007; 67:735-741.

20. Kan MW, Leung LH, Yu PK. The performance of the progressive resolution optimizer (PRO) for RapidArc planning in targets with low-density media. Journal of 
applied clinical medical physics/American College of Medical Physics. 2013; 14:4382.

21. Li Y, Rodrigues A, Li T, Yuan L, Yin FF, Wu QJ. Impact of dose calculation accuracy during optimization on lung IMRT plan quality. Journal of applied clinical medical physics/American College of Medical Physics. 2015; 16:5137.

22. Kan MW, Cheung JY, Leung LH, Lau BM, Yu PK. The accuracy of dose calculations by anisotropic analytical algorithms for stereotactic radiotherapy in nasopharyngeal carcinoma. Physics in medicine and biology. 2011; 56:397-413.
23. Kan MW, Leung LH, Yu PK. Dosimetric impact of using the Acuros XB algorithm for intensity modulated radiation therapy and RapidArc planning in nasopharyngeal carcinomas. International journal of radiation oncology, biology, physics. 2013; 85:e73-80. 